\title{
Jakarta, Bangkok, Rio de Janeiro
}

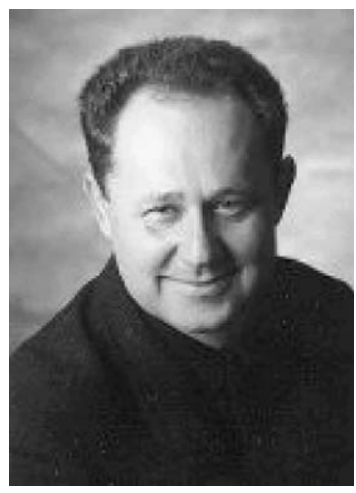

Prof. Dr. med. Manfred Wildner

Bibliografie

DOI http://dx.doi.org/

10.1055/s-0032-1321792

Gesundheitswesen 2012;

74: 405-406

(c) Georg Thieme Verlag KG

Stuttgart · New York

ISSN 0941-3790

\section{Korrespondenzadresse}

Prof. Dr. med. Manfred Wildner

Bayerisches Landesamt für Gesundheit und Lebensmittelsicherheit

Veterinärstraße 2

85762 Oberschleißheim

manfred.wildner@Igl.bayern.de
Rio de Janeiro, Bangkok, Jakarta - Orte, von denen wir träumen. Als Städteurlauber, Weltenbummler oder Badegäste, an den Stränden von Ipanema und der Copacabana beispielsweise liegend, idealerweise braungebrannt und mit perfekten Köpermaßen. Allerdings bleiben solche Träume häufig „kontrafaktisch“, die Realität sieht doch anders aus. Vielleicht erreichen wir immerhin die Strände von Grado oder der Costa Brava, wenn auch mit einem Body-Mass-Index im hochnormalen Bereich. Und träumen dort weiter.

Die Fähigkeit zur Vorstellung kontrafaktischer Welten, dessen, was noch nicht oder nicht mehr ist, was sein könnte oder - je nach psychischer Grundstruktur - sein sollte, kann uns dabei dennoch eine Leichtigkeit des Seins bescheren: ein Wert an sich. Möglicherweise eine Triebfeder der Evolution? Die Verhaltensforscherin Alison Gopnik ist der Frage nach der Fähigkeit zur kontrafaktischen Vorwegnahme von Möglichkeiten und Handlungsoptionen im Kleinkindes- und Kindesalter nachgegangen, mit faszinierendem Ergebnis: schon Kleinstkinder scheinen in der Lage zu sein, sich imaginäre Möglichkeiten fernab der tatsächlichen Verhältnisse vorzustellen und entsprechende Handlungen bzw. Äußerungen zu vollziehen [1]. Kulturgeschichtlich sei diesbezüglich auch an den persischen Arzt und Philosophen Ibn Sina (lat. Avicenna, 980-1037 n.Chr.) erinnert. In einem berühmten Gedankenexperiment träumte er sich aus seinem Kerker hinausschwebend in die Freiheit: Für ihn der Beweis einer vom Körper unabhängig existierenden Seele. „Cogito, ergo sum“ (,ich denke, also bin ich“), formulierte später René Descartes, wobei das Denken als neurophysiologischer Vorgang in heutigem Verständnis auch das (Tag-)Träumen umfasst.

Der Bezug zum Gesundheitswesen? Kontrafaktische Herangehensweisen sind bewährte und effektive Triebfedern für das „Not-wendige“ geduldige Arbeiten im therapeutischen und rehabilitativen Bereich. Diese Anstrengungen sind angesichts von Krankheits- und Unfallfolgen oftmals getragen von Erinnerungen an die in der Vergangenheit schon einmal erlebte umfänglichere funktionale Gesundheit. Diese Erinnerungen motivieren sowohl Patientinnen und Patienten wie auch Therapeuten [2]. Im Feld von Prävention und Gesundheitsförderung ist für die Motivationen eine interessante Umkehr der zeitlichen Kausalität zu beobachten. Die Ursache für präventives Handeln im hier und heute liegt oftmals in einer vorweggenommenen besseren Zukunft: Diese Zukunft, die es noch nie gab, wirkt motivierend „Zurück“ in die Gegenwart („Zielursache“).
Beispiele solcher rückwärtswirksamen Zukunftsentwürfe sind die Gesundheitsdefinition der WHO von Gesundheit als Zustand vollständigem körperlichen, seelischen und sozialen Wohlbefindens, sind wissenschaftlich-(nano)technologische „Science Fiction“ in Literatur, Fachliteratur und Förderanträgen und sind gesellschaftliche Utopien wie ein „Recht auf Gesundheit“. U-topos: das, was (noch) keinen Platz in unseren überkommenen und selbstgeschaffenen Realitäten hat. Ein Beispiel einer solchen Utopie in der Sozialmedizin ist die wissenschaftsbasierte Utopie des „System(s) einer vollständigen medizinischen Polizey“ Johann Peter Franks im 18. Jahrhundert [3,4]. Ausprägungen des 21sten und vorweggenommenen 22 . Jahrhunderts solcher medizinischer Utopien und wissenschaftsbasierter Fiktionen sind die Genomik und andere „omic“-Wissenschaften einer prädiktiven, personalisierten, präventiven, partizipatorischen „Präzisions“-Medizin, auch für die Bevölkerungsgesundheit [5-8]. Dabei seien die Begriffe Utopie und Fiktion wertneutral verstanden, als durchaus mögliche Entwürfe eines wissenschaftlich denkbaren und gesellschaftlich lohnenswerten Fortschritts.

Häufig sind in Hinblick auf Gesundheit Städtenamen mit kontrafaktischen Zukunftsentwürfen verbunden. Sie reichen von der Erklärung von Alma Ata (1978), welches ein Menschenrecht auf Gesundheit, die Bedeutung der wirtschaftlichen und sozialen Entwicklung sowie der Primärversorgung betont, über die Ottawa-Charta zur Gesundheitsförderung (1986) mit einer Vielzahl von Folgekonferenzen (Adelaide 1988, Sundsvall 1991, Jakarta 1997, Mexico 2000, Bangkok 2005, Nairobi 2009 und - geplant - Helsinki 2013), zu spezifischen Themen wie Fragen der menschlichen Fortpflanzung und reproduktiver Menschenrechte (Kairo 1994), zu den verbindenden Werten europäischer Gesundheitssysteme (Tallinn 2008) und zu verbesserter globaler Gouvernanz für mehr und gerechter verteilte Gesundheit (Rio de Janeiro 2012). Was diese Städte auch symbolisieren: Gesundheit ist zunehmend global und in ihrer geografischen Lokalisation spiegeln sich auch Veränderungen der globalen politischen Landschaft.

Skizzieren derartige Städtenamen Landkarten der Hoffnung oder sind es lediglich geistige Fluchtpunkte, Exilorte einer oftmals als bedrückend erlebten Realität? Vielleicht beides, in einem produktiven Wechselspiel. Die rechtliche Bindung derartiger Chartas und Deklarationen ist in der Regel schwach. Ihr Hauptnutzen liegt in der Legitimation von Veränderungsvorschlägen 
und zugehörigen Reformbewegungen. Diese können sich ggf. auf derartige Chartas und Deklarationen abstützen und sind damit prinzipiell eingebunden in den nationalen und internationalen gesundheitlichen Zukunftsdiskurs. Diese Ansätze als Utopien zu belächeln mag allenfalls kurzfristig gerechtfertigt sein. Hat nicht beispielsweise die Allgemeine Erklärung der Menschenrechte trotz ihrer rechtlichen Form als Deklaration über die Jahre und Jahrzehnte doch eine hohe Durchsetzungskraft und formgebende Kraft bewiesen, wie auch die Ottawa-Charta zur Gesundheitsförderung? Und sind nicht auch unsere rechtlich stärker bewährten Bürgerlichen, Straf- und Sozialgesetzbücher in gewissem Sinn ebenfalls gesellschaftliche Utopien, Idealvorstellungen des Miteinanders?

Auch die bewährten quantitativen, statistischen wie epidemiologischen, Methoden bedienen sich dieser Kontrafaktizität: In Form des „was wäre, wenn“ von kontrollierten randomisierten Studien, von nach Exposition stratifizierter Kohorten, Hoch- und Vorausberechnungen in Modellen unterschiedlicher mathematischer Spezifizierungen [9]. Die Fähigkeit zu kontrafaktischen Vorstellungen als Bedingung wissenschaftlich-technischer Aufbrüche? Als „Prinzip Hoffnung“ der Weg aus einer existenziellen Angst, hin zu einer erst zu schaffenden wahrhaft menschlichen Heimat [10]? Doch Vorsicht: haben nicht gerade weitgreifende soziale Utopien auch unfassbare Angst und unfassbares Elend erzeugt, von GULag-Systemen bis zu Pol Pot? Sind nicht Visionen zugleich auch mit Risiken behaftete Spekulationen? Vielleicht täte im gesellschaftspolitischen Raum eine Pflicht zu Modellprojekten mit selbstkritischer, redlicher objektiver Evaluation und Diskussion vor jeder weiter reichenden Gesetzgebung gut. Für die forschende Wissenschaft gilt dies allemal.

Auch die Beiträge in dieser Ausgabe stellen wieder, teilweise mit kontrafaktischen Elementen, dringlich nachgefragte Erkenntnisse für unsere gesellschaftlichen und wissenschaftlichen Orientierungs- und Gestaltungsaufgaben vor und damit zur Diskussion: Zur Arbeit des Ärztlichen Zentrums für Qualität in der Medizin, zu den Herausforderungen einer alternden Gesellschaft im ländlichen Bereich und zu regionaler Deprivation und Mortalität, zur Gebietswahl von Medizinstudierenden und zur sozialmedizinischen Lehre sowie zu den Personbezogenen Faktoren der ICF.

Interessant ist, dass zumindest in der jüdisch-christlich geprägten Kulturhistorie am Anfang ein (verlorener) Paradiesgarten als ideale natürliche Umwelt steht (Genesis, Kap.2, 1-25), am Ende das himmlische Jerusalem, also eine ideale Stadt (Offenbarung des Johannes, Kap. 21,11-15). Diese ist in den Texten durchaus konstruktiv-architektonisch aussagekräftig beschrieben, als von würfelförmiger Gestalt, strahlend von Licht und mit einer Kantenlänge von 12 mal 1000 Stadien. In heutigen Längenmaßen und in mathematischer Sprache wären dies nüchterne $2220 \mathrm{~km}$ mit daraus ableitbarem Rauminhalt und vermutlich ebenfalls ökologisch ableitbarer maximaler Bewohnerzahl (siehe z.B. Biosphere2, http://www.b2science.org/). In einer Deutung als symbolische Sprache wiederum wäre 12 die Zahl der Ganzheit, 1000 die Zahl der Fülle und eine Berechnung der maximalen Einwohnerzahl gar nicht intendiert. Die Leser mögen ihre sie überzeugende Interpretation dieser Utopie wählen. Was die Interpretationen einigt, ist die Vorstellung von Stadt als Ort des menschlich vorteilhaft Geschaffenen, dem Menschen Entsprechenden und ihn auch in der sozialen Interaktion Tragenden. Welche Chancen bieten sich daraus z.B. für Heidelberg, Berlin, München? Noch sind mit diesen Städten keine international rezipierten Deklarationen aus Medizin oder Gesundheitswissenschaften verbunden. Also einerseits: nur zu. Andererseits mag das Fehlen von mit diesen Städtenamen verbundenen Erklärungen auch gar kein Nachteil sein. Gerade die noch nicht ausformulierten Träume laden ein zum zukunftsoffenen paradiesischutopischen gedanklichen Spazieren, Probieren - oder einfach träumend am (Urlaubs-)Strand Liegen. Kontrafaktische Ideen entfalten auch so ihre Kraft.

\section{Literatur}

1 Gopnik A. Kleine Philosophen: Was wir von unseren Kindern über Liebe, Wahrheit und den Sinn des Lebens lernen können. Berlin: Ullstein Verlag; 2009

2 Bauby JD. Schmetterling und Taucherglocke. Wien: Paul Zsolnay Verlag; 1997

3 Pieper A. Johann Peter Frank: Vom Arzt zum Gesundheitspolitiker. Dtsch Arztebl 2003; 100 (28-29): A-1951/B-1618/C-1526

4 Frank JP. System einer vollständigen medicinischen Polizey. Mehrbändiges Werk. URL http://resolver.sub.uni-goettingen.de/purl?PPN333 511387 Zugriff am 06.06.2012

5 Mirnezami R, Nicholson J, Darzi A. Preparing for precision medicine. N Eng J Med 2012, doi:10.1056/NEJMp1114866

6 Pang T, Weatherall $D$. Genomics and world health: a decade on. Lancet 2012; 379: 1853-1854

7 Hood L, Friend SH. Predictive, personalized, preventive, participatory (P4) cancer medicine. Nature Reviews Clinical Oncology March 2011; 8: $184-187$

8 Brand A, Schröder P, Brand H, Zimmern R. Getting Ready for the Future: Integration of Genomics into Public Health Research, Policy and Practice in Europe and Globally. Community Genet 2006; 9: 67-71

9 Stang A. Kausalität und Confounding in der Epidemiologie. Gesundheitswesen 2011; 73: 884-887

10 Bloch E. Werkausgabe: Band 5: Das Prinzip Hoffnung. Suhrkamp; Frankfurt am Main: 1985 